\title{
The value of observational study data in healthcare decision making: An Indian perspective
}

Although efficacy and safety data from randomized clinical trials (RCT) is considered to be the key to evidence generation, it is no longer adequate to meet all needs of Indian healthcare decision makers. Supplementing RCT data with robust observational studies data (OSD) as a decision making tool is being increasingly used by many payer advisors. Health Technology Assessment (HTA) agencies and healthcare bodies meaningfully use OSD for numerous purposes including, to confirm results of RCTs, justify utility estimates in economic models and demonstrate durability of effects of healthcare interventions. The phenomenal growth of Indian healthcare field has necessitated the need for robust HTA involving RCT data and OSD for better decision making. OSD may come a long way in supplementing Indian stakeholders in making rational healthcare decisions. It will be interesting to see how frequently OSD will be utilized in the Indian healthcare sector, and also how meaningfully, in the future.

Key words: Health technology assessment, healthcare decision making, observational study, real world evidence

\section{Amit Dang, T. A. Manjunath}

MarksMan Healthcare Solutions, Navi Mumbai, 'ESIS Hospital, Mumbai, Maharashtra, India

Address for the Correspondence: Dr. Amit Dang, MarksMan Healthcare Solutions, $1^{\text {st }}$ Floor, Plot No. 6, Sector 12A, Kopar Khairane, Navi Mumbai, Maharashtra 400709, India.

E-mail: amit.d@ marksmanhealthcare.com

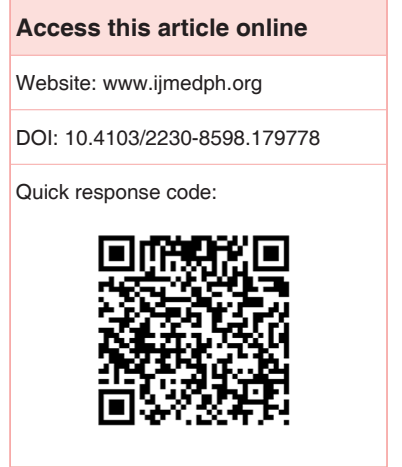

Health technology assessment (HTA) is a multidisciplinary activity that systematically examines the safety, clinical efficacy and effectiveness, cost, cost-effectiveness, organizational implications, social consequences, legal and ethical considerations of the application of a health technology - usually a drug. ${ }^{[1]}$ Observational studies contribute to developing evidence, understanding existing disparities in health care access, as well as making a rational selection and ensuring proper delivery of healthcare services. While there is a growing demand for information about health care decision-making, there is very little understanding about when observational studies are appropriate for decision support.

Observational studies are conducted to study the casual effects of certain agents, procedures, treatments or programs or when the investigator cannot perform a controlled experiment. ${ }^{[2]}$ Three main study designs are used in observational studies namely cohort, case-control, and cross-sectional. ${ }^{[3]}$ Cohort studies follow patients who are exposed to interventions to determine the incidence of the outcome. Several study outcomes can be studied within the same study in this design. Relative risk and incidence rate ratios are the most common frequency measures assessed in a cohort study. ${ }^{[3]}$ A case-control study identifies individuals (cases) who have the outcome of interest and controls who do not. The exposure is compared between the cases and the controls. A case-control study estimates a relative frequency measure called the odds ratio, which is an estimate of the relative risk. It is useful for studying rare outcomes, which would require a huge sample size in a cohort design. They are generally cheaper and simpler. A cross-sectional study (also known as prevalence studies) measures the exposure and outcome simultaneously, although the temporal association between

This is an open access article distributed under the terms of the Creative Commons AttributionNonCommercial-ShareAlike 3.0 License, which allows others to remix, tweak, and build upon the work non-commercially, as long as the author is credited and the new creations are licensed under the identical terms.

For reprints contact: reprints@medknow.com

How to cite this article: Dang A, Manjunath TA. The value of observational study data in healthcare decision making: An Indian perspective. Int J Med Public Health 2016;6:53-5. 
them is not possible. ${ }^{[4]}$ Cross-sectional studies are generally used to provide the basis for a subsequent cohort or case-control studies or a randomized controlled trial (RCT).

The usual notion that exists among the researchers and healthcare policy makers is that clinical RCTs are the "gold standard" for healthcare evaluations and observational study methods viz., cohort and case-control studies have little or no value. ${ }^{[5]}$ It is unfortunate to note the remarks of the eminent medical epidemiologist Richard Doll that observational study methods "provide no useful means of assessing the value of therapy." ${ }^{[6]}$ This remark superficially denotes the attitude of clinicians and healthcare workers toward the data obtained employing observation research methods.

Observational studies are an inexpensive way to give the real world scenario and provide information about diverse populations, practitioners, and settings in a timely and cost-effective manner. They often employ simpler study designs and are easy to carry out. The use of different types of study methods can yield different types of data, both qualitative as well as quantitative. Observational study data (OSD) can complement other approaches, augment existing evidence, and can even link together data sets that offer a wealth of information about real-world interventions and outcomes. ${ }^{[7]}$

In actual fact, OSD helps payers make well-informed and rational health care decisions. Supplementing randomized clinical trial data with robust OSD are a crucial decision-making tool that is being increasingly used by payer advisors in many parts of the world.

Liden et al., ${ }^{[8]}$ evaluated decisions of HTA agencies worldwide including the UK's National Institute for Health and Clinical Excellence, Scotland's Scottish Medicines Consortium (SMC), Canada's Canadian Agency for Drugs and Technologies in Health Common Drug Review and Pan-Canadian Oncology Drug Review, Australia's Pharmaceutical Benefits Advisory Committee (PBAC), France's Haute Autorité de Santé (HAS), and Germany's The Federal Joint Committee, as when they used observational studies in HTA decisions. A total of 1840 HTA decisions were reviewed. Decisions were classified as either "positive" or "negative." Only $106(6 \%)$ HTA evaluations incorporated OSD and of these $77 \%$ resulted in positive decisions. Of the HTA evaluations that did not consider observational data, only $67 \%$ resulted in a positive decision $(P=0.025)$.

Several HTA decisions have been based on observational studies. For example, the PBAC (Australia) evaluated Yervoy Ipilimumab for treatment of patients with Stages III and IV malignant melanoma in July 2011. PBAC did not recommend the drug based on the submitted RCT data, noting that, although it demonstrated improved efficacy, it had an inferior safety profile and issues with costs and eligible patient population. On the third submission in 2012, Bristol-Myers Squibb presented postregistration data of Yervoy's effect. Citing this OSD, PBAC recommended Yervoy..$^{\left[{ }^{[]}\right.}$
In Scotland, OSD helped Zaltrap (aflibercept) demonstrate improvement in quality of life (QoL). SMC reviewed Zaltrap in combination with FOLFIRI chemotherapy for the treatment of adults with metastatic colorectal cancer in 2003. SMC evaluated RCT and despite demonstrating significantly longer overall survival, Zaltrap was not recommended as it lacked a robust economic analysis. In the 2014 resubmission, Sanofi submitted OSD from two open-label studies assessing safety and QoL. ${ }^{[10]}$ This OSD was used to revise the utility values within the economic model. SMC recommended Zaltrap because the new data demonstrated a substantial improvement in QoL.

In 2010, HAS approved Myozyme for the treatment of late-onset Pompe disease in France using evidence generated by OSD. An observational study of 44 patients, ${ }^{[11]}$ data from the French Pompe disease registry and RCT data was submitted. The drug improved the 6-min walk test and seated vital capacity in the observational study.

HTA agencies and healthcare bodies meaningfully use OSD for numerous purposes including, to confirm results of RCTs, justify utility estimates in economic models and demonstrate durability of effects of healthcare interventions. HTA promises to improve patient care and medical technologies, taking into consideration of the social, economic, and clinical nuances. Properly implemented, striking a clear balance between the economic and ethical perspectives, HTA has a major role to play in healthcare decisions in Indian healthcare sector. OSD may come a long way in supplementing Indian stakeholders in making rational health care decisions and is likely to depict a real world picture. It will be interesting to see how frequently OSD will be utilized in the Indian healthcare sector, and also how meaningfully, in the future.

\section{Financial support and sponsorship}

Nil.

\section{Conflicts of interest}

There are no conflicts of interest.

\section{REFERENCES}

1. Battista RN, Hodge MJ. The evolving paradigm of health technology assessment: Reflections for the millennium. CMAJ 1999;160:1464-7.

2. Cochran WG. The planning of observational studies of human populations (with discussion). J R Stat Soc 1965;128:134-55.

3. Jepsen P, Johnsen SP, Gillman MW, Sørensen HT. Interpretation of observational studies. Heart 2004;90:956-60.

4. Concato J, Shah N, Horwitz RI. Randomized, controlled trials, observational studies, and the hierarchy of research designs. N Engl J Med 2000;342:1887-92.

5. Black N. Why we need observational studies to evaluate the effectiveness of health care. BMJ 1996;312:1215-8.

6. Doll R. Summation of the conference. Ann $N$ Y Acad Sci 1993;703:310-3.

7. Fleurance RL, Naci H, Jansen JP. The critical role of observational evidence in comparative effectiveness research. Health Aff (Millwood) 
2010;29:1826-33. Available from: https://www.rtihs.org/sites/default/files/ Hogue_ISPOR_May2013.pdf. [Last accessed on 2015 Jul 25].

8. Liden D, Jaksa A, Yin Ho. Does Real World Evidence Matter in Health Technology Assessments? Published in Pharmaphorum on 27 January, 2015. Available from: http://www.pharmaphorum.com/articles/ does- real-world-evidence-matter-in-health-technology-assessments. [Last accessed on 2015 Jul 25].

9. Wonder M. Wonder Drug Consulting Pty Ltd. Reimbursement success rates and timelines for new medicines for cancer; an international comparison; 3 February, 2014. Available from: http://medicinesaustralia.
com.au/files/2013/07/140323_OIT_Wonder-Report_FINAL.pdf. [Last accessed on 2015 Aug 07].

10. Sobrero A, Bordonaro E, Frassineti L. Utility assessment of health related quality of life in metastatic colorectal cancer patients: Interim analyses of the global Aflibercept safety and HRQoL Program, ESMO; October, 2013. p. 162.

11. Strothotte S, Strigl-Pill N, Grunert B, Kornblum C, Eger K, Wessig C, et al. Enzyme replacement therapy with alglucosidase alfa in 44 patients with late-onset glycogen storage disease type 2: 12-month results of an observational clinical trial. J Neurol 2010;257:91-7. 